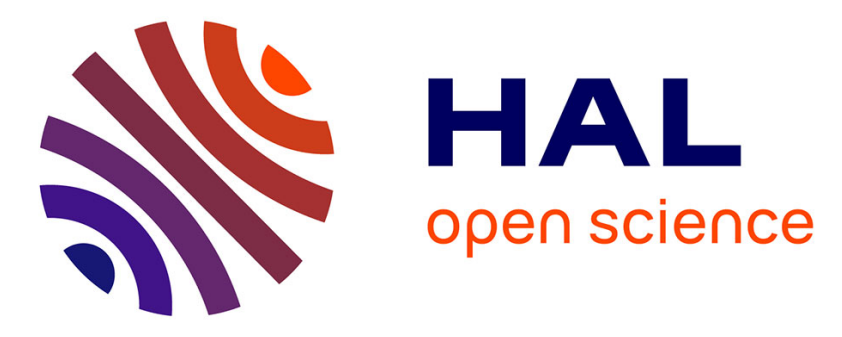

\title{
Influence of the stitching density on the transverse permeability of Non-Crimped New Concept (NC2) multiaxial reinforcements : measurements and predictions
}

\author{
Sylvain Drapier, Alexandre Pagot, Alain Vautrin, Patrick Henrat
}

\section{To cite this version:}

Sylvain Drapier, Alexandre Pagot, Alain Vautrin, Patrick Henrat. Influence of the stitching density on the transverse permeability of Non-Crimped New Concept (NC2) multiaxial reinforcements: measurements and predictions. Composites Science and Technology, 2002, 62 (15), pp.1979-1991. 10.1016/S0266-3538(02)00127-6 . emse-00563475

HAL Id: emse-00563475

https://hal-emse.ccsd.cnrs.fr/emse-00563475

Submitted on 5 Feb 2011

HAL is a multi-disciplinary open access archive for the deposit and dissemination of scientific research documents, whether they are published or not. The documents may come from teaching and research institutions in France or abroad, or from public or private research centers.
L'archive ouverte pluridisciplinaire HAL, est destinée au dépôt et à la diffusion de documents scientifiques de niveau recherche, publiés ou non, émanant des établissements d'enseignement et de recherche français ou étrangers, des laboratoires publics ou privés. 


\title{
Influence of the stitching density on the transverse permeability of Non-Crimped New Concept (NC2) multiaxial reinforcements : measurements and predictions
}

\author{
S. Drapier ${ }^{\dagger} 1$, A.Pagot ${ }^{\dagger \star}$, A. Vautrin ${ }^{\dagger}$, and P. Henrat ${ }^{\dagger}$ \\ ${ }^{\dagger}$ Mechanical and Materials Engineering Department - SMS Division \\ École Nationale Supérieure des Mines de Saint-Étienne \\ 42023 Saint-Étienne Cedex 02, France \\ Tel : (+33)(0)4 77420079 Fax : (+33)(0)4 77420249 \\ E-mail : drapier@emse.fr, vautrin@emse.fr \\ †^ now at Institut Français du Pétrole \\ 1 et 4 , avenue de Bois-Préau \\ 92852 Rueil-Malmaison Cedex, France \\ E-mail : alexandre.pagot@ifp.fr \\ $¥$ Hexcel Fabrics \\ ZI Les nappes - 38630 Les Avenières \\ Tel : (+33)(0)4 74338860 Fax : (+33)(0)4 74339902 \\ E-mail : patrick.henrat@hexcel-fabrics.fr
}

\begin{abstract}
New manufacturing processes arise for polymer-based composites which involve resin infusion through dry pre-forms. Modelling approaches of these processes require to assess new physical characteristics of the materials. In this paper, the transverse permeability of new multiaxial stitched materials, referred to as $N C 2$, is investigated. First, in the framework of Darcy's flows, this permeability is assessed for various biaxial NC2 using a specific device. Through this approach it is shown that the transverse permeability depends strongly on the stitching density. Then, the results from a simplified FE study carried out at the blanket scale are shown to correlate quite well the experimental measurements and evidences established in the first part.
\end{abstract}

\footnotetext{
${ }^{1}$ to whom correspondence should be addressed
} 


\section{Keywords}

multiaxial reinforcements, through-thickness stitching, resin infusion, transverse permeability, mesoscopical approach

\section{Introduction}

Till the early 90's, high performance organic composite structures were mainly manufactured from pre-impregnated unidirectional plies. Since then, processes involving the resin impregnation in dry pre-forms have become very popular due to their versatility along with the cost reductions that they usually induce. Indeed, valuable cost cuts originate from the handling and storage cost reductions, since only the resin has to be cold-stored. But moreover in these processes thick/heavy multilayered preforms can be easily employed, this in turn induces material costs reductions.

In these manufacturing processes the impregnation stage is a key parameter ([15], [2]). Originally, liquid resins were injected through injection gates in molds containing the dry preforms. The most popular of these manufacturing processes, widely used, is the Resin Transfer Molding (RTM). Modelling efforts, still continued, have permitted to optimise for the RTM the injection gates and events placements in the molds ([14], [12]), in order for the mold to be completely filled and free of any air bubble which may be entrapped [15]. This injection takes place mainly in the preform plane and hence involves principally the longitudinal permeabilities properties of the preforms ([22], [19], [3]). Other processes, involving rather resin infusion than resin injection and referred to as Liquid Composite Molding ( $L C M$ ), have been developed in the very last few years. LCM processes permit to overtake these problems of injection heterogeneities by impregnating the dry preforms over wide surfaces [11]. In this sense, lower pressures are involved, and the transverse permeability properties of the preforms become one of the key parameters to optimize the manufacturing cycles.

One of these processes is the Resin Film Infusion ( $R F I)$ process in which films of neat resin are stacked, with dry preforms, in a mold. The use of a movable rigid perforated plate, or molds for more complex shapes, placed on top of the stacking permits to ensure well-finished final surfaces. The whole stacking is then vacuum-bagged and usually 
placed in an autoclave to undergo a temperature-pressure cycle (Figure 1). It is clear that the filling time and resin heterogeneity distributions in this process will depend mainly on the preform through-thickness permeability. This parameter is very seldom studied, since conversely to the longitudinal permeabilities, the length over which it can be measured is very small, of the order of $1 \mathrm{~mm}$ for single standard multiaxial reinforcements.

The present paper is dedicated to the study of the transverse permeability in long-fibre composites, more precisely in new multiaxial semi-products developed by Hexcel Fabrics. These Non-Crimped New Concepts (NC2) multiaxial reinforcements are made up from very homogeneous planes of fibres stitched together through their thickness (see Figure 2). The very high resulting homogeneity, essential to achieve high final mechanical properties, leads to very low permeabilities, much lower than the very few ones measured on other more classical materials. Then, in order to optimize the pressure-temperature cycles, the transverse permeability of single $N C 2$ multiaxial fabrics must be input in some modelings of the impregnation process at the fabric scale.

In the present study, first, based on a quick survey of permeability measurements and fluid propagation in porous media, a specific set-up is designed to measure properly this permeability. This set-up is then used to assess the through-thickness permeability of biaxial NC2. These basic multiaxial fabrics are considered here to isolate the phenomena controlling the permeability. Then some simulations are presented at the blanket scale which focus on the effect of the stitching density onto the permeability. A good correlation is found between experimental measurements and predicted permeabilities regarding the stitching density influence.

\section{The permeability phenomenon in composites}

The transverse permeability phenomenon across composite multiaxial fabrics is introduced here, this permits to set up the basis of the principle of measurements. Then, longitudinal and transverse permeability measurements are presented.This yields some ideas for the set-up to be designed for the transverse permeability characterization. Finally, since a modelling approach is proposed in the second part of this paper, some representative models are presented which set the basis for the simulations. 


\subsection{Flow through porous media}

The multiaxial fabrics studied here are intrinsically porous media, the pore size of which may be at the fibre level as well as at the stitching level (Figure 2). The basics of flows through porous media can be found for instance in [13]. In these flows, the interconnected porosity is involved which restrains, at the microscopic scale, the fluid progression through 'channel-like' cavities. Hence, at this scale the classical laws of fluid mechanics will rule the flow. The two main laws commonly used are the mass conservation equation and the Navier-Stokes equation, provided the fluid can be assumed as newtonian. When compared to a classical mass conservation equation, the volume fraction of any phase must be accounted for. Let us notice that in a wet fabric, the phases are either fluid for the resin in motion, or solid for the composite which can undergo deformation under the pressure generated by the flow. For this latter, the mechanical response must be determined too.

These two laws permit on their own to describe the fluid flow through the porous medium, where pressure and velocity are the parameters to determine in both phases. However, beyond the modelling efforts required for seeking the solution, the complexity of the porosity architecture does not allow in our case this system to be solved easily. Even numerically, the various porosity shapes which exist at the different scales cannot be easily represented without turning to more refined descriptions such those adopted in [10] or [7] for example. In the present approach, these equations will be solved through fluid mechanics finite element (FE) analysis in a simplified framework, following the experimental evidences demonstrated in the measurements.

\subsection{Assessing permeabilities from flows in composites}

In porous media, such as the fabrics of interest in composites, more convenient approaches can be led at the mesoscopic scale, i.e. the scale where the porous medium is seen as homogeneous. Several approaches exist which are used in the literature to model or to identify the permeability. The most popular approach relies on the Darcy's law, empirically established, which permits to relate in three dimensional cases the flow velocity to 
the gradient of pressure undergone by the fluid in the porous medium :

$$
\mathbf{v}=-\frac{\widetilde{\mathbf{K}}}{\eta} \nabla P
$$

where $\mathbf{v}$ is the flow rate vector, $\widetilde{\mathbf{K}}$ is the second order permeability tensor of the porous medium that usually depends on the fluid flowing through it represented here by its viscosity $\eta$, and $\nabla P$ is the pressure gradient. However, it must be pointed out that this empirical law has been established in a well defined frame. Indeed, the Darcy's law holds only for stationary regimes, and Reynolds $(R e)$ numbers calculated for porous media must be lower than 1. Beyond this limit, the flow is inertial $(1<R e<150)$, and further $(300<R e)$ turbulent. This Reynolds number maybe somehow hard to evaluate in porous media since it depends on a characteristic length of the medium [13], which in turn depends on the scale of observation considered.

For the experimental measurements the simplistic Darcy's approach is conveniently considered, either for longitudinal ([1], [10], ...), transverse ([22],[3]), or 3D permeability characterization [5]. Along with this flow rule, a usual restriction is made to the second order permeability tensor that is taken to be orthotropic. Moreover, it is assumed that no coupling exists between the permeabilities in the three principal directions. Finally, as a first approximation only three independent constants define the permeability tensor : two in-plane (longitudinal) permeabilities and one (through-thickness) transverse permeability. For uniaxial flows, a simplified form is deduced from Eq. (1) which relates the mean uniaxial velocity of the fluid to the pressure gradient undergone in the flow direction (for instance $\mathbf{e}_{3}$ ). The pressure gradient is here assumed to be linear, since it is deduced from the discrete measurements of the pressure loss along the flow. Moreover, it is assumed that the transverse direction is, in first approximation, a principal direction. Then, for an orthotropic medium, the uniaxial expression writes for a transverse flow :

$$
D=-\frac{K_{33} A}{\eta} \frac{\Delta P}{e}
$$

where $D$ is the flux, $K_{33}$ is the transverse permeability, $A$ is the cross-sectional area of the flow, $\Delta P$ is the pressure variation, $\eta$ is the fluid viscosity, and $e$ is the flow length. All these quantities refer to the flow direction. In our case this approach will be used provided the permeability properties can depend on multiaxial architecture.

As for the NC2 material investigated here, it must be noticed that scientific papers devoted to permeability measurements of multiaxial composites mainly focus on woven or 
random reinforcements ([3], [10], [6]), [16], ...). However, in some points multiaxial NC2 and woven fabrics have a close meso-structure, especially both exhibit an alternance of fibre tows and gaps. This meso-structure is important in setting the channels through which the resin will flow, either in injection or infusion. But this is mostly relevant in longitudinal permeability measurements. For the transverse permeability in NC2 materials, macropores are present due to the stitching. Consequently, the fluid will certainly flow through these channel-like connections and then continue either in the fabric planes or in the closest stitching upstream. This will be assessed in the second part dedicated to the transverse permeability modelling.

\subsection{Longitudinal and transverse measurements}

Permeability measurements can be carried out for either in-plane (for instance [22], [19], [18], [17], [3], ...), transverse ([3] and [22], ...) or 3D flows ([5]), in unsteady or steady regimes. The principle of measurement is common for both longitudinal and transverse measurements and is rather simple. A specific device is fed in with fluid under constant flux or pressure. Meanwhile pressure or velocity measurements are made in the dry (downstream) and wet (upstream) regions, on either side of the flow front (Figure 3-a), or specimen location (3-b) in saturated regime. Permeability is then identified, using the Darcy's law (Eq. 2 or 1) or more complex relationships in bidirectional or 3D cases, from the variations of velocity or pressure induced by the displacement of the fluid through the sample tested. For longitudinal measurements, rectangular or circular specimens are cut, respectively for unidirectional and bidirectional measurements, and fitted into a rigid mold.

For transverse measurements there is no such restrictions on the specimen shape, but usually circular cylindrical specimens are more conveniently used. This transverse measurement is very seldom studied ([22], [5], [3] ... ) but tends to be more and more common due to the use of thicker fabrics. The very key point is in this case to realize the fluid injection while maintaining the specimen to be impregnated, but without disturbing the fluid flow. In [3] specimens are maintained on their edges, but this does not allow to control the specimen thickness. Therefore the fibre volume fraction, defined as the ratio of the fibre volume over the tested volume, could vary since the apparent fabric thickness may change. It must be pointed out that in the transverse permeability measurement, the 
fibre volume fraction is of great importance since it will influence the fabric permeability, i.e. will change the porosity configuration. In [22], the thickness can be controlled by compacting the specimen between 2 porous rigid plates allowing for the injection. The counterpart of this technological solution is that the pressure loss induced by the plate porosity may be higher than the one induced by the specimen itself, due to the reduced thickness involved. In either case, the measurement takes place in saturated regime since it is not possible to visualise the flow front over such a small length.

\subsection{Through-thickness permeability predictions}

As stated previously, commonly permeability phenomena in composites rely on the treatment of their complex meso/micro-structure. Then idealized geometries are widely used, either to predict permeabilities in basic cases [13], or in an attempt to relate experimental permeabilities to micro, meso and macro scales [19]. In more refined approaches, such as the lattice Boltzmann model proposed by [20], phenomena can be represented at the mesoscopical scale whilst dealing with void formation in the tows. Results of transverse flows simulations over circular porous tows are proposed which describe the void entrapment in higher permeability regions for unsaturated flows. In our composite materials, saturated flow will be simulated, and consequently any entrapment will be ignored.

This qualitative analysis [20] applies also to more realistic pore architecture, the complexity of which has to be addressed if quantitative predictions are to be proposed. In the case of composites ([10], [21]), starting from micrographs the identification of pores at micro and meso scales is deduced from the probabilistic treatment that a segment of length $l$ fits in a pore in a given direction. For soils [7], the pore network is reconstructed from 3D image analyses, after skeletization and creation of a spatialized local pore size distribution. Then the 'conductivity' of the network is evaluated, especially fluid invasion is shown to be controlled by the connectivity between the largest pores. In our materials, where at least two pore scales exist, such a result reinforces our choice of modelling the flow at the mesoscopical scale where the largest pores are seen.

Eventually, homogenization methods exist which are the most relevant regarding the permeability of media with a regular micro/meso-structure ([23], [4]). These methods rely on the behaviour of a representative volume element on which are prescribed boundary conditions ensuring its periodicity. In our case, $N C 2$ fabrics cannot be easily represented 
through a succession of elementary cells at the microscopical level. However, the idea of using a representative volume element can be adapted to the simulation of transverse permeability at the mesoscopical scale. Eventually, finite element approaches are in our case the most easily suitable for the predictions of transverse permeability using a representative mesoscopical cell, and will be subsequently used.

\section{Assessment of the $N C 2$ transverse permeability}

Using the knowledge gained in the previous survey, an apparatus has been designed to measure the transverse permeability of fabrics. This experimental device is presented and then used to assess the $N C 2$ transverse permeability, and further to demonstrate how this permeability varies with the stitching density.

\subsection{Experimental apparatus}

The principle of measuring the pressure loss induced by the flow of the fluid through the specimen is the basis of the permeability measurement. The specific apparatus developed here is based on this principle (Figure 4 and 5). Following [22], aluminium honeycombs are selected to maintain the specimen in the apparatus. Indeed, after some FE simulations, the channels formed naturally by the honeycomb structure were shown to make the flow become steady even for high fluid velocities. Hence, the use of honeycombs will allow for the thickness of the tested volume to be controlled while smoothing the flow. The second key point of the apparatus is the pressure measurement which is realized with two pressure probes. Let us notice that the location of the probes along the flow is optimized such that pressures are measured in steady flow regions before and after the honeycombs (Figure 4-b).

Another very important point, as explained previously, is the porosity volume fraction of the tested medium that will depend on the compression required to maintain the specimen. Conversely to the processes involving resin injection, the reinforcement deformation during impregnation appears very rarely in infusion processes [24]. In our case, the specimen thickness will be controlled during the measurements with integrated verniers, and 
the porosity will be deduced from the specimen surfacic mass. Furthermore, the paper of [17], dedicated to the repeatability measurements for longitudinal permeability, states that some discrepancies of the order of $15 \%$ may result from heterogeneities in the material as well as from the material deformability. The deformability of the specimen will especially take place during the cutting. Following this evidence, the specimen cutting is integrated to our apparatus such that specimens will not be handled at all (Figure 4-a and Figure 5-b).

This in-situ cutting implies that the airtightness, required both to prevent the fluid from leaking and to isolate the chambers, has to be mobile. This is achieved in the presented apparatus as shown in Figure (4-b) and Figure (5-a). For further simplifications, the specimens tested were chosen circular, $100 \mathrm{~mm}$ in diameter. The specimen diameter was fixed such that for the lowest stitching density $(10 \mathrm{~mm} \times 10 \mathrm{~mm})$, statistically at least $96 \%$ of the stitching points would be submitted to the flow for any random placement of the specimen.

Eventually, for the impregnating fluid a lubrication oil NEUTRAL was selected, the behaviour of which is newtonian. The oil was chosen such that its viscosity was as close as possible to the resin viscosity encountered in the RFI process, taking into account that the gear-pump used for the feeding (Figure 4-b) would not stand fluid viscosities larger than 10 Pas. The oil viscosity is perfectly known as a function of temperature, and is given in Annexe 1. The fluid temperature is evaluated through a thermo-couple placed between the pump and the fluid inlet, and any apparent thickness variation is assessed using an LVDT displacement sensor. An interface-card is used for data acquisition on a PC, for pressures, temperature, displacement, and flow rate.

\subsection{Protocol}

Measurements are carried out with the presented apparatus (Figure 5). The flow length related to the fibre volume fraction of the specimen is set by the verniers, close to commonly used values. As for the velocity range, it is rather hard to know exactly the velocities encountered in the manufacturing processes. Hence, in order to cover the wider velocity range, it was chosen to stop to increase the fluid velocity (flux) when the upper actuator used to maintain the upper chamber was no longer able to sustain the pressure induced by the flow across the fabric. The pressure applied for maintaining the system closed is 7.5 
bars, similar to the pressure which is used in the industrial RFI process.

The following protocol was used for the measurements. A multiaxial fabric is placed on the lower honeycomb and the upper mobile part comes down in contact with the verniers. The vibrating cutting system is turned on and maintained until the blade has completed the cut (Figures 4-a and 5-b). The airtightness mobile system can then move upward to ensure both the absence of air in the fluid flow and leaks out of the chambers (Figures 4b). In the first times of the cycle, a low fluid velocity is prescribed to the servo-gear pump which feeds in the apparatus with fluid. In this stage the lower chamber is filled, and then the fabric is impregnated. Once the second pressure probe can measure the fluid flow, the servo-gear pump is stopped, and then a complete test cycle can be started in which the fluid velocity is increased step by step. Every step is continued until pressures stability is reached.

\subsection{Assessment of the transverse permeability}

\subsubsection{Permeability measurement}

A typical measurement is presented in Figure 6 for a biaxial $[0,90]$ NC2 made up from $2 \times$ Tenax HTS5131 $267 \mathrm{~g} / \mathrm{m}^{2}$ carbon fabrics stitched $10 \mathrm{~mm} \times 10 \mathrm{~mm}$. In this figure the resistance provided by the fabrics against the flow, i.e. the pressure gradient deduced from the pressure loss and flow length $e\left(\frac{\Delta P}{e}\right)$ over the fluid viscosity $\eta$, is plotted as a function of the fluid velocity (v). It is verified that the largest Reynolds number of the flow encountered is lower than 1 :

$$
R e^{\max }=\frac{\rho v^{\max } d}{\eta}=1.75 \cdot 10^{-2}<<1
$$

with $\rho$ the volumic mass of the oil $\left(\rho=877 \mathrm{~kg} \mathrm{~m}^{-3}\right), v^{\max }$ the maximum fluid velocity encountered $\left(v^{\max } \leq 3 \cdot 10^{-3} \mathrm{~m} . \mathrm{s}^{-1}\right), d$ is the largest characteristic length of the NC2 taken as the largest stitching hole diameter measured from micrographs $(d \leq 0.4 \mathrm{~mm})$ and $\eta=0.06$ Pa.s is the fluid viscosity at room temperature. Darcy's approach is then valid for these measurements. Then, simply by using the Darcy's law for uniaxial flows (Eq. 2), the transverse permeability $\left(\mathrm{K}_{33}\right)$ can be identified from these data.

One will notice that this resistance of the fabric dos not depend linearly on the fluid velocity as one would expect. Conversely, in other more 'rigid' materials tested, such as woven 
fabrics and metallic lattices, a linear relationship is observed between the resistance and the fluid velocity. Therefore, this non-linearity seems to reflect some intrinsic properties of the NC2 materials. Further studies are currently under progress to clarify this point. However, one wishes to make a productive use of the data given by this first approach of the NC2 transverse permeability. Consequently, the nominal transverse permeability is assessed as the permeability prevailing at the onset of flowing in the NC2 (Figure 6). At that stage the fibre volume fraction is perfectly controlled and the meso-structure is not disturbed. Eventually, for the present study, the nominal apparent permeability will be identified as the inverse of the initial slope (Figure 6).

\subsubsection{Measurement repeatability}

The assessment of measure repeatability has been achieved for 2 types of biaxial $N C 2$ [0,90], made up of identical UD Tenax fabrics $267 \mathrm{~g} / \mathrm{m}^{2}$ (HTS 5131 / 24k tows), but with different stitching pattern. The first biaxial was stitched with a $10 \mathrm{~mm} \times 10 \mathrm{~mm}$ pattern and the other one with a $5 \mathrm{~mm} \times 5 \mathrm{~mm}$ pattern, both were stitched with PA 6.678 tex stitching yarn. The largest discrepancies are for the $10 \mathrm{~mm} \times 10 \mathrm{~mm}$ stitching pattern. The secant apparent permeability, deduced from the current slope or a given point on the resistance curve, reach about $20 \%$ of variation (Figure 7) for the fluid velocity tested. However, one must point out that the work of [17], dedicated to the repeatability measurements for longitudinal permeability reports some discrepancies of the order of $15 \%$, but for longitudinal flows over several hundreds of millimeters $(300 \mathrm{~mm})$. In our case, transverse measurements are made over some lengths of the order of one millimeter or less. Moreover, transverse permeabilities are of the order of $10^{-13} \mathrm{~m}^{2}$, whereas longitudinal permeabilities are at least one decade higher, of the order of $10^{-12} \mathrm{~m}^{2}$.

\subsection{Influence of the stitching density}

The transverse permeability of $N C 2$ is assessed with the presented dedicated apparatus, using the protocol introduced. Essentially, biaxial materials are tested, all of them made up from the same Tenax HTS 5131 UD dry fabrics. The stitching, when reported, is realized with a PA 6.678 tex stitching yarn. This kind of biaxial material permit to reveal many specificities of $N C 2$ multiaxial fabrics without involving too much complexity 
related to tricky stacking sequences or architectures.

Assessing the influence of the stitching onto the transverse permeability is the main issue of the present work. To achieve this, 6 types of biaxial fabrics were tested. First $[0]_{2}$ and $[0,90]$ fabrics, both unstitched, were tested. Then, biaxial $[0,90] N C 2$, made up of the same fabrics were manufactured but with 3 different balanced stitching patterns of $10 \mathrm{~mm} \times \mathrm{mm}, 5 \mathrm{~mm} \times 5 \mathrm{~mm}$, and $2.5 \mathrm{~mm} \times 2.5 \mathrm{~mm}$, corresponding respectively to stitching densities of $10^{4}, 4.10^{4}$, and $16.10^{4}$ stitches per $\mathrm{m}^{2}$. Eventually, biaxial [+45,-45] $N C 2$ with a $5 \mathrm{~mm} \times 10 \mathrm{~mm}$ stitching pattern were tested. Since the same UD blankets are used, the fibre volume fraction is set to 0.65 for all the materials tested.

First, permeabilities for the $[0]_{2}$ and $[0,90]$ unstitched biaxial are very close (Figure 6). Unfortunately, the very low permeability (of the order of $2 \cdot 10^{-14} \mathrm{~m}^{2}$ ) of these UD fabrics may, under a 7.5 bars closure pressure, cause leaks in the apparatus even for a moderate flux. Therefore, only few flow rates can be tested but which are sufficient to draw some clear trends about these measurements. The curves obtained for both unstitched materials tend to coincide. The similarity between these material responses demonstrates that the resulting transverse permeability does not depend at all on the stacking sequence. At the opposite, the introduction of stitching $(10 \mathrm{~mm} \times 10 \mathrm{~mm})$ seems to increase greatly the permeability which jumps from $0.24 \cdot 10^{-13} \mathrm{~m}^{2}$ to $1.1 \cdot 10^{-13} \mathrm{~m}^{2}$.

This strong influence is confirmed by the curves for the other material tested (Figure 8) which clearly show that the permeability largely depends upon the stitching. But first, it must be pointed out that the differences between theses experimental results are much higher than the repeatability of the measures themselves. Therefore, these variations can be interpreted as intrinsically related to the material tested. More precisely, this permeability can even be related directly to the stitching density only since actually the independence of the transverse permeability upon the stacking sequence is again confirmed by the $[+45,-45] N C 2$ measurement (Table 1). Indeed, in that material stitched $5 \mathrm{~mm} \times 10 \mathrm{~mm}$, only the stitch density seems to control the permeability comprised between the permeabilities for the biaxials $[0,90]$ stitched $10 \mathrm{~mm} \times 10 \mathrm{~mm}$ and $5 \mathrm{~mm} \times 5 \mathrm{~mm}$.

The stitching density seems to control the transverse permeability, this is clearly illustrated in Figure 9 where the permeability is shown to vary, linearly in first approximation, with the stitching density. In fact, mesoscopical measurements of the fabric geometry re- 
veal that only the holes left around the stitching pitches are open pores, the size of which are quite comparable for the various fabrics tested $(\sim 0.4 \mathrm{~mm})$. In first approximation this suggests that mainly these spaces will allow for the fluid to pass through. It can be easily reckoned that permeability depends on the amount of surface spaces for the fluid to flow, directly related to the stitching density. One will notice that, although the gap geometry is not exactly the same for the $[+45,-45]$ biaxial $N C 2$ as for $[0,90]$ (see in Figures 2-a and 2-b), the effective (open pores) surface spaces may depend essentially on the stitching yarn itself.

From these results, it can be concluded that the stitching density is of prime importance regarding the transverse permeability of $N C 2$. Although this stitching is introduced mainly to improve the handability and the mechanical properties of the resulting structural component, it appears to be also very helpful in increasing the permeability. Indeed, with a stitching density of $10^{4}$ pts. $\mathrm{m}^{-2}$, the transverse permeability is increased by a factor of 5 with respect to the same unstitched biaxial. By increasing further the stitching density by 16 (from $10 \times 10 \mathrm{~mm}$ to $2.5 \mathrm{~mm} \times 2.5 \mathrm{~mm}$ ), a similar increase is reached for the permeability. Regarding the very low permeability of unstitched materials, this increase of the permeability is essential in achieving proper manufacturings. There is obviously a limit in increasing the stitching density since the mechanical counterpart is that a decrease in the material properties is expected due to the geometrical imperfections resulting from the introduction of stitches, as it can be observed at larger amplitudes in NCF materials ([8], [9]).

\section{Permeability predictions}

The aim of this simulation is to model the influence of the stitching density onto the transverse permeability. The Ansys FE package is used to model the permeability response at the mesoscopical level, on a representative volume element of the biaxial $[0,90]$ fabric tested previously. 


\subsection{Representative volume element}

FE simulations are carried out on the biaxial $N C 2$ characterized experimentally. In order to avoid very demanding computations, we use the principle of homogenization techniques based on repeating representative volume elements (RVE). It is assumed that the response of this volume element is representative of the response of the entire medium, provided proper boundary conditions are prescribed on its edges to represent neighbouring volume elements.

For the modelling, the scale of observation has to be chosen first. The choice of this scale must permit to grasp the main features which may influence the permeability. For this choice, first one can notice that there is the formation of inter-tow spaces (gaps) (Figure 2 ), independently on the direction of the stitching with respect to the fibre orientation. These gaps are similar to the ones developing in $N C F$ manufactured with the Liba process for instance, but with a minor amplitude. Secondly, the other main features which may influence the transverse permeability are the stitches that were shown to strongly control the permeability (Figure 9). Lastly, as saturated flow simulations are considered here, any air entrapment in the tows can be neglected. Consequently, it seems natural to set up the model at the mesoscopical scale where gaps and stitches can be accounted for, and where tows are seen as homogeneous porous media. Therefore, in our biaxial material a RVE is easily extracted, the planar dimensions of which are equal to the stitching pattern used (Figure 10) and its thickness corresponds to the fabric thickness. By applying proper boundary conditions, detailed below, the permeability of the equivalent infinite fabric can be computed.

\subsection{Computations}

For the sake of simplicity, the chosen RVE has been idealized. By symmetry, only a quarter of this RVE must be represented. Also, gaps between the tows induced by the stitching yarns (see Figure 2) are represented as parallelepipeds (Figure 11-a), resulting from the space left between the planes standing for the 0 and $90^{\circ}$ tows (Figure 11-b). For the stitching, a space is considered as the union of the space left by the circular cylindrical stitching and the gap between the tows (Figure 11-a). Stitching densities are represented by varying the cell dimensions $\left(\mathrm{p}_{1}, \mathrm{p}_{2}\right)$ corresponding to the stitching pitch respectively 
along $\mathbf{e}_{1}$ and $\mathbf{e}_{2}$ directions.

The inter-tow gaps and the stitch hole are modeled with fluidic elements in which the Navier-Stokes equation prevail. For modelling tows, i.e. the porous medium, the Darcy's law is employed instead of the previous laws. Both media are meshed with quadratic isoparametric finite elements. Let us notice that in every article where permeability is simulated for a reinforcement, tows are supposed not to be permeable. However, due to the fabric homogeneity, in our case macropores are much less numerous. It is then possible that flow takes place through the tows as it can be observed in the RTM process [3]. Tow permeability is defined through a diagonal tensor. This is what prevented us from computing the $[+45,-45]$ biaxial NC2 permeability. The transverse permeability of the tows is the one assessed for a UD fabric $\left(2.2510^{-14} \mathrm{~m}^{2}\right)$, and in the fibre direction it is taken equal to $10^{-12} \mathrm{~m}^{2}$ following the estimations from [17]. As for the fluid viscosity, it is equal to the viscosity of the lubrication oil used for experiments measured at room temperature $(\eta=0.06$ Pa.s) .

As can be seen in Figure 11 the stitch hole is not represented, it is instead modeled with zero normal flux conditions, i.e. no fluid can penetrate the stitching yarn but the flow along this yarn is allowed. Indeed, implicitly it is assumed that the stitching yarn induces a hole, the diameter of which $(0.3 \mathrm{~mm})$ corresponds exactly to the yarn diameter deduced from its linear mass and its density. The other boundary conditions are zero normal flux conditions on the lateral faces of the cell. To represent the pressure drop induced by the flow through the fabric, a 1 bar pressure is applied on the upper face of the RVE while on the lower face the pressure is null. Permeability is calculated through the Darcy's law (Eq. 2) from both the pressure differential prescribed and the mean fluid velocity deduced from the massic flux computed.

\subsection{Results - Comparison with experiments}

Permeabilities are computed for [0,90] fabrics considering the 3 stitching densities tested. The results reported in Table 2 are also plotted in Figure 12 where can be seen the good correlation between the predicted permeabilities and the ones measured. The maximum discrepancy of about $25 \%$ is reached for the highest stitching densities (4 and $16.10^{4}$ pts. $\left.\mathrm{m}^{-2}\right)$, whereas for the lowest density it is $8 \%\left(1 \cdot 10^{4} \mathrm{pts} . \mathrm{m}^{-2}\right)$. 
The most important effect confirmed by these computations is the effect of the stitching density on the permeability. The simulations reveal that the fluid flows through the fabric (Figure 13) only across the stitching holes. Indeed, whatever the stitching density simulated, the flow rate of fluid across the tows remains low. The tows are so little permeable that the fluid cannot flow across and passes almost in totality through the stitching holes. This is illustrated by the distribution of the transverse fluid velocity plotted in Figure 13. Transverse velocities in the tows are 2 decades lower than the transverse velocities in the stitching hole. More particularly the fluid circulate in the narrow region between the gaps and the stitching yarn.

Assuming that the stitching pitches remain large enough, the interaction between flows in the stitching cylinders is negligible. Then, one can propose a very simple relationship that would relate the transverse permeability for a given stitching density $d_{1}\left(\mathrm{~K}_{33}^{d 1}\right)$ to the permeability of the same fabric but with a stitching density $d_{2}\left(\mathrm{~K}_{33}^{d 2}\right)$ (Eq. 4). Predictions with this very basic relationship compare well with the FE results (Table 2).

$$
K_{33}^{d 1}=K_{33}^{d 2} \frac{d_{1}}{d_{2}}
$$

As a conclusion, both experimentally and theoretically, it is established that the fluid flows almost exclusively through the stitching holes. Some discrepancies exist between predicted and measured permeabilities, however regarding the very low permeabilities identified these moderate differences are acceptable for current industrial applications. Then, as a rule of thumb, assuming that the stitching hole geometry and the gap size will not depend on the stitching density, a linear relationship exist between the transverse permeability and the stitching density. For industrial applications, stitching densities are at least 10000 pts. $\mathrm{m}^{-2}$ but will usually not exceed 40000 pts. $\mathrm{m}^{-2}$. This ensures that flow across the tows may be negligible, and that in turn this linear approximation will hold.

\section{Conclusion}

The first study of the influence of the stitching density on the transverse permeability of $N C 2$ was carried out. The main mechanisms which control the flow of a fluid across $N C 2$ were assessed. Eventually, predictions were shown to compare well with experimental 
results.

First, a specific apparatus was designed to measure the transverse permeability in multiaxial fabrics. The key point of this system is to maintain properly the specimen, using aluminium honeycombs, while ensuring the fibre volume fraction control. Also, the specimen cutting is integrated to the apparatus which ensures that the fabric deformability will not yield any meso-structure variations. This apparatus allows to evaluate the transverse permeability through the uniaxial Darcy's law, knowing both pressure loss induced by the specimen resistance to the fluid flow, and fluid velocity.

Experiments were carried out on 6 different fabrics, both unstitched and stitched, and with several stacking sequences and stitching patterns. Experimentally it was demonstrated that neither the stacking sequence nor the stitching pattern would influence the transverse permeability. Conversely, the stitching density was shown to control greatly the permeability. Indeed, changing the stitching pitch from $10 \mathrm{~mm} \times 10 \mathrm{~mm}$ to $2.5 \mathrm{~mm} \times 2.5 \mathrm{~mm}$, i.e. increasing by 16 the stitching density, permits to increase the permeability by about the same amount, from $1.1 \cdot 10^{-13} \mathrm{~m}^{2}$ to $15.1 \cdot 10^{-13} \mathrm{~m}^{2}$. From a manufacturing point of view, this improvement of the transverse permeability ensures a good fibre impregnation, and consequently optimum final mechanical characteristics.

This main result was found also by the FE simulations carried out at the mesoscopical scale on a representative volume element of $[0,90]$ biaxial fabrics. Permeabilities were assessed by measuring the flux as a response to a differential of pressure applied to the fluid crossing the porous media standing for the tows. It was established that mainly the fluid flowed along the stitching yarn, in the stitching holes. Then, in first approximation, it is the surfacic ratio of spaces left by the stitching points which controls the permeability. This provides a clear explanation to the experimental dependence of the transverse permeability upon the stitching density. Eventually, as a first approximation, the permeability for any admissible stitching density can be extrapolated linearly from a single permeability known for a given stitching density.

Based on the knowledge gained here, this permeability study should be extended to tri and 
quadri-axial $N C 2$. For the measurements, these materials will be tested with the apparatus developed here. As for the simulations, some problem may arise due to the non-diagonal terms of the permeability tensor which will appear for non cross-plies.

Acknowledgements The authors wish to acknowledge the Rhône-Alpes Region for its support to this work through the programme 'Thématique Prioritaire 2000-2002'. 


\section{Annexe : fluid viscosity}

The fluid used, a lubrication oil (NEUTRAL), appeared to vary in viscosity with the ambient temperature. The characterization of this viscosity over a wide range of temperatures yields the following results (Table An. 1 and Figure An. 1).

\begin{tabular}{|c|c|c|c|c|c|c|c|c|c|}
\hline Temperature $\left(T\right.$ in $\left.{ }^{\circ} \mathrm{C}\right)$ & 5 & 15 & 18 & 20 & 22 & 25 & 30 & 40 & 50 \\
\hline Viscosity $\left(\eta\right.$ in $10^{-3}$ Pa.s $)$ & 239 & 113.7 & 93.2 & 82 & 72.5 & 60.7 & 46 & 28 & 18.3 \\
\hline
\end{tabular}

Table An. 1 : Oil viscosity $v s$. temperature

This viscosity can be fitted by the following fourth degree polynomial (Eq. 5), leading to at most $1.1 \%$ of discrepancy, and $0.44 \%$ in the range $15^{\circ} \mathrm{C}-25^{\circ} \mathrm{C}$ of interest here (Figure An. 1):

$$
\eta(T)\left(10^{-3} \text { Pas }\right)=9.6955 E^{-6} T^{4}-1.4803 E^{-2} T^{3}+0.88167 T^{2}-25.782 T+3.4761
$$

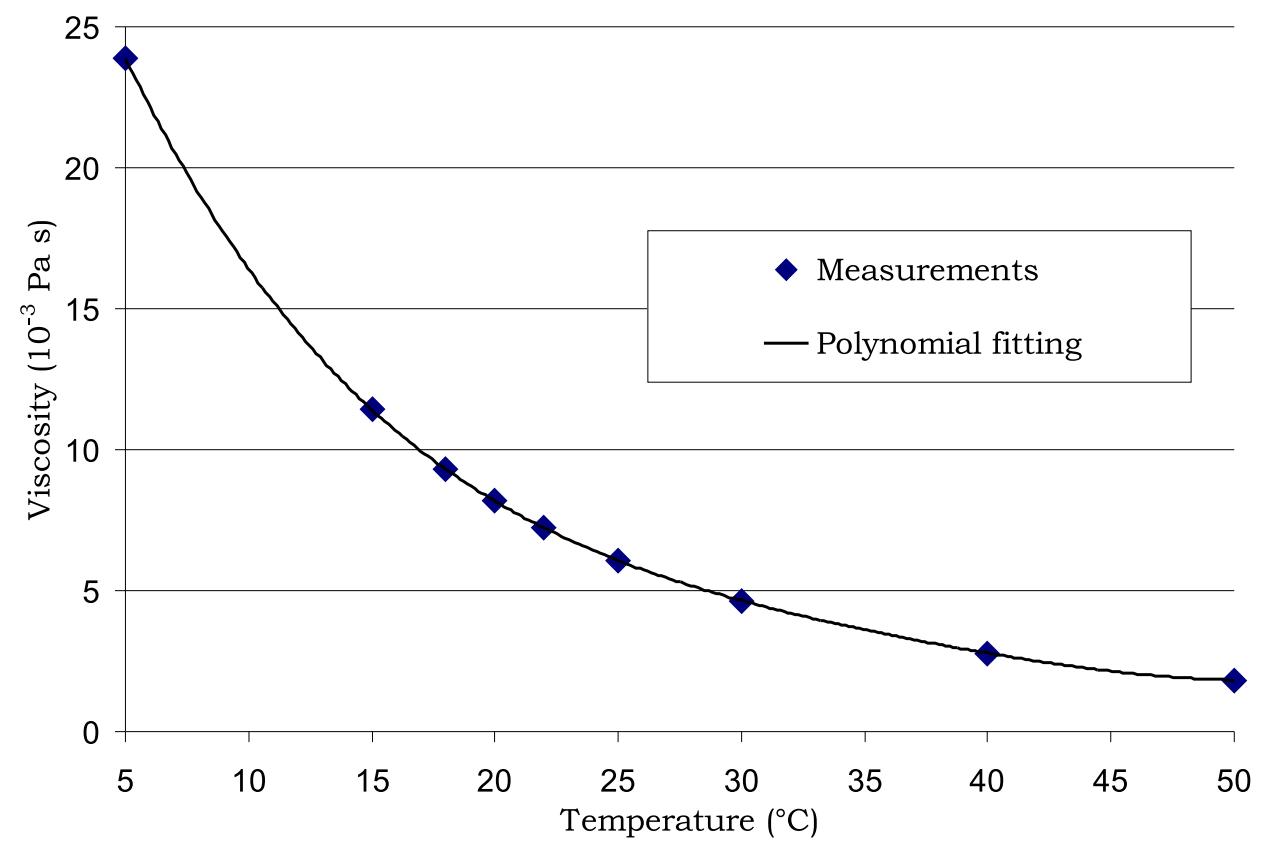

Figure An. 1 : Variation of the oil viscosity vs. temperature. 


\section{References}

[1] K.L. Adams, B. Miller, and L. Rebenfeld. Forced in-plane flow of an epoxy resin in fibrous networks. Polym. Eng. Sci., 26(20):1434-1441, 1986.

[2] R. Avila and F.L. Matthews. An experimental investigation of the isothermal impregnation of fibrous preforms. In Institute of Material Composite Division, editor, Proceedings of ECCM9, Brighton 4-7 June 2000, 2000.

[3] C. Binétruy and J. Pabiot. Effects of fabrics architectural heterogeneities on effective and satured permeabilities in RTM. In T. Massard and A. Vautrin, editors, Proceedings of ICCM12 - Paris 5-9 July 1999, number 178, page 682, 1999.

[4] C. Boutin. Study of the permeability by periodic and self-consistent homogenisation. Eur. J. Mech. A/Solids, 19:603-632, 2000.

[5] J. Bréard, A. Saouab, and G. Bouquet. Mesure de la perméabilité spatiale d'un renfort tridimensionnel pour matériaux composites à matrice polymère. Eur. Phys. J. AP, 1:269-278, 1998.

[6] A.C. Caba, R.B. Rattazzi, and A.C. Loos. Verification of a simulation model for resin film infusion of complex shaped composite structures. J. Rein. Plast. Comp., 18(16):1465-1477, 1999.

[7] J.F. Delerue, E. Perrier, Z.Y. Yu, and B. Velde. New algorithms in 3d image analysis and their application to the measurement of a spatialized pore size disctribution in soils. Pys. Chem. Earth (A), 24(7):639-644, 1999.

[8] S. Drapier and M.R. Wisnom. Finite-element investigation of the compressive strength of non-crimp-fabric-based composites. Comp. Sci. Technol., 59(12):12871297, 1999.

[9] S. Drapier and M.R. Wisnom. Finite-element investigation of the interlaminar shear strength of non-crimp-fabric-based composites. Comp. Sci. Technol., 59(16):23512362, 1999.

[10] Y. Henzel, J. Bréard, F. Trochu, and R. Blanlot. A standard characterisation of satured and unsatured flow behaviours in porous media. In T. Massard and A. Vautrin, editors, Proceedings of ICCM12 - Paris 5-9 July 1999, number 604, page 690, 1999. 
[11] S.C. Joshi, X.L. Liu, Y.C. Lam, and J. Sheridan. Simulation of resin film infusion process using finite element / nodal control volume approach. Advanced Composite Letters, 8(3):101-104, 1999.

[12] M.K. Kang, J.J. Jung, and W.I. Lee. Analysis of resin transfer moulding process with controlled multiple gates resin injection. Composites / A, 31:407-422, 2000.

[13] S. Kaviany. Principle of heat transfert in porous media. Springer-Verlag New York Inc., 1991.

[14] J.M. Kenny, A. Trivisano, and G. Molina. Mathematical modelling of resin transfer molding of composites for automotive applications. In A. Miravette, editor, Proceedings of ICCM9 - Madrid July 1993, volume 3, pages 513-515. Woodhead Publishing Ltd., 1993.

[15] Y.C. Lam, S.C. Joshi, and X.L. Liu. Numerical simulation of the mould-filling process in resin-transfer moulding. Comp. Sci. Technol., 60(6):845-855, 2000.

[16] S.T. Lim, M.K. Kang, and W.I. Lee. Modelling of void formation during resin transfer molding. In T. Massard and A. Vautrin, editors, Proceedings of ICCM12 Paris 5-9 July 1999, number 627, page 544, 1999.

[17] T.S. Lundström, R. Stenberg, R. Bergström, H. Partanen, and P.-A. Birkeland. Inplane permeability measurements : stability, repatability and reproductibility. In T. Massard and A. Vautrin, editors, Proceedings of ICCM12 - Paris 5-9 July 1999, number 309, page 696, 1999.

[18] F. Robitaille, A.C. Long, B.J. Souter, and C.D. Rudd. Permeability modelling of industrial preforms : simulations and practical aspects. In Institute of Material Composite Division, editor, Proceedings of ECCM9, Brighton 4-7 June 2000, 1999.

[19] C.-H. Shih and L.J. Lee. Effect of fiber architecture on permeability in liquid composite molding. Polym. Compos., 19(5):626-639, 1998.

[20] M.A.A. Spaid and F.R.Jr Phelan. Lattice boltzmann methods for modeling microscale flow in fibrous porous media. Phys. fluids, 9(9):2468 - 2474, 1997. 
[21] V. P. Stavrov, E.I. Kremenevskaya, V. V. Stavrov, and V.M. Tkachev. Effect of the structure of a fibrous layer on its permeability for a nonlinearly viscous fluid. Mech. of Comp. Mat., 33(4):554-563, 1997.

[22] L. Trevino, K. Rupel, W.B. Young, M.J. Liou, and L.J. Lee. Analysis of resin injection molding in molds with preplaced fiber mats : Permeability andd compressibility measurements. Polym. Compos., 12(1):20-29, 1991.

[23] K. Velten. Drag measurement and evaluation in impregnation processes. In Institute of Material Composite Division, editor, Proceedings of ECCM9, Brighton 4-7 June $2000,1999$.

[24] T.J. Walsh, C.T. Morse, and O.O. Ochoa. Composite auto bumper : design and process evaluation. In A. Miravette, editor, Proceedings of ICCM9 - Madrid July 1993, volume 6, pages 252-259. Woodhead Publishing Ltd., 1993. 


\section{List of Tables}

1 Transverse permeability measurements for the 6 materials tested. . . . . . 24

2 Transverse permeability predicted and measured for the 4 stitched biaxial materials tested. . . . . . . . . . . . . . . . . . . . 24 


\begin{tabular}{|l|l|l|l|l|l|l|}
\hline Material & $\begin{array}{l}{[0]_{2}} \\
\text { unst. }\end{array}$ & $\begin{array}{l}{[0,90]} \\
\text { unst. }\end{array}$ & $\begin{array}{l}{[0,90]} \\
10 \times 10\end{array}$ & $\begin{array}{l}{[0,90]} \\
5 \times 5\end{array}$ & $\begin{array}{l}{[0,90]} \\
2.5 \times 2.5\end{array}$ & $\begin{array}{l}{[ \pm 45]} \\
5 \times 10\end{array}$ \\
\hline \hline Stitching density $\left(10^{4}\right.$ pts.m $\left.{ }^{-2}\right)$ & - & - & 1 & 4 & 16 & 2 \\
\hline$K_{33}$ measured $\left(10^{-13} \mathrm{~m}^{2}\right)$ & 0.21 & 0.24 & 1.1 & 6.37 & 15.1 & 2.47 \\
\hline
\end{tabular}

Table 1: Transverse permeability measurements for the 6 materials tested.

\begin{tabular}{|l|l|l|l|l|}
\hline Material & $\begin{array}{l}{[0,90]} \\
10 \times 10\end{array}$ & $\begin{array}{l}{[ \pm 45]} \\
5 \times 10\end{array}$ & $\begin{array}{l}{[0,90]} \\
5 \times 5\end{array}$ & $\begin{array}{l}{[0,90]} \\
2.5 \times 2.5\end{array}$ \\
\hline \hline Stitching density $\left(10^{4} \mathrm{pts} . \mathrm{m}^{-2}\right)$ & 1 & 2 & 4 & 16 \\
\hline$K_{33}$ computed $\left(10^{-13} \mathrm{~m}^{2}\right)$ & 1.19 & - & 4.75 & 19.1 \\
\hline Predictions/Measurements & $-8 \%$ & - & $-25 \%$ & $27 \%$ \\
\hline \hline$K_{33}$ analytical (Eq. 4) $\left(10^{-13} \mathrm{~m}^{2}\right)$ & 1.19 & 2.38 & 4.76 & 19.1 \\
\hline
\end{tabular}

Table 2: Transverse permeability predicted and measured for the 4 stitched biaxial materials tested. 


\section{List of Figures}

1 Schematic of the Resin Film Infusion process tooling. . . . . . . . . . . 27

2 Picture of a quadriaxial $N C 2([-45,0,45,90])$ made up of dry carbon UD fabrics (Tenax HTS $5131 / 24 \mathrm{k}$ tows $150 \mathrm{~g} / \mathrm{m}^{2}$ ), stitching pitch $5 \mathrm{~mm} \times 5 \mathrm{~mm}$ : (a) $-45^{\circ}$ face and (b) $90^{\circ}$ face. . . . . . . . . . . . . . . . . . 27

3 Schematic of (a) longitudinal, and (b) transverse permeability measurements. . . . . . . . . . . . . . . . . . . 28

4 Schematic of the through-thickness permeability measurement device, in the 2 main stages of the protocol : (a) cutting stage, and (b) measurement stage. . . . . . . . . . . . . . . . . . . 29

5 Apparatus designed for transverse permeability measurements : (a) description, (b) cutting stage prior to measurements . . . . . . . . . . . 30

6 Resistance of fabrics to the fluid flow versus the fluid velocity for fabrics $2 \times 267 \mathrm{~g} / \mathrm{m}^{2}:[0]_{2}$ unstitched, [0,90] unstitched, and [0,90] stitched

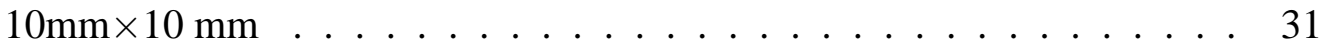

7 Secant permeability measurements $\left(K_{33}^{s}\right)$ versus the fluid velocity (v) for a biaxial $[0,90] N C 22 \times 267 \mathrm{~g} / \mathrm{m}^{2}$ stitching pitch $10 \mathrm{~mm} \times 10 \mathrm{~mm} \ldots \ldots 32$

8 Resistance to the fluid flow versus the fluid velocity for fabrics $2 \times 267$ $\mathrm{g} / \mathrm{m}^{2}:[0]_{2}$ unstitched, biaxial $[0,90]$ unstitched and stitched $10 \mathrm{~mm} \times 10 \mathrm{~mm}$, $5 \mathrm{~mm} \times 5 \mathrm{~mm}$, and $2.5 \mathrm{~mm} \times 2.5 \mathrm{~mm}$. . . . . . . . . . . . . . . 33

9 Permeability of the materials tested $v$ s their stitching density. . . . . . . . 34

10 Representative Volume Element for the biaxial $N C 2$ permeability simula-

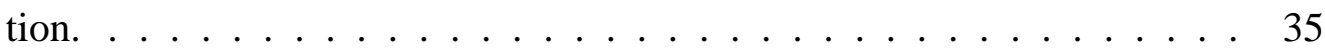

11 RVE mesh used for FE simulations : (a) mesh of the gaps with fluidic elements, (b) mesh of the tows with solid porous elements, and (c) complete mesh of the RVE. . . . . . . . . . . . . . . . . . 36

12 Permeability predicted and measured, for the materials tested, $v s$ their stitching density. ................... 37 
13 Distribution of the transverse fluid velocity $\left(V_{3}\right)$ in $m \cdot s^{-1}$ for the $[0,90]$ NC2 $5 \mathrm{~mm} \times 5 \mathrm{~mm}$ stitching pitch. . . . . . . . . . 38 


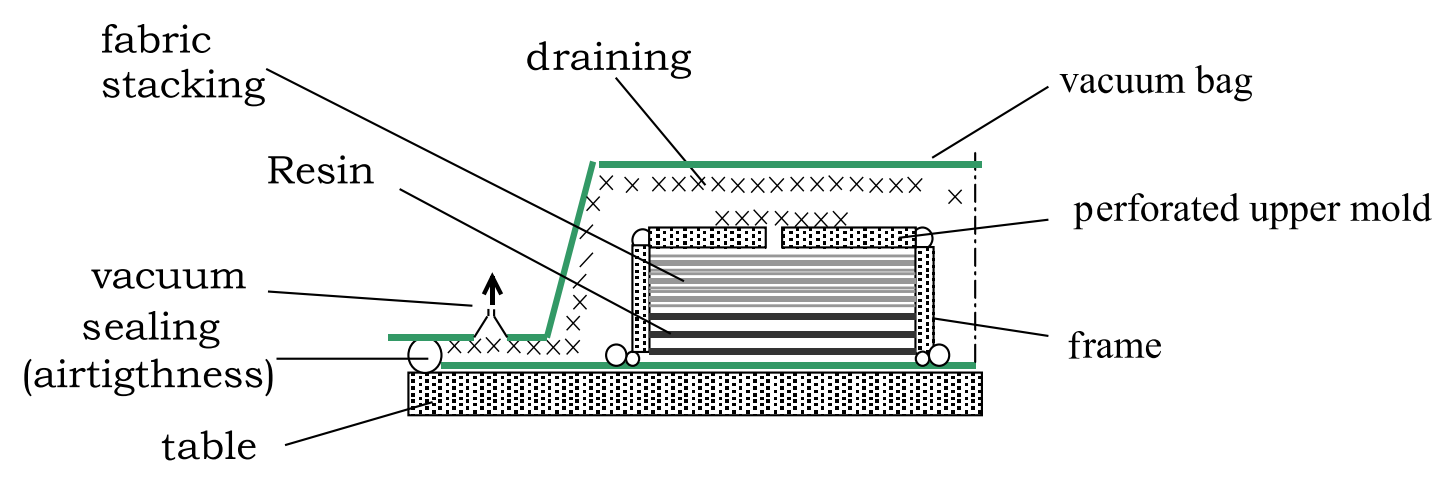

Figure 1: Schematic of the Resin Film Infusion process tooling.

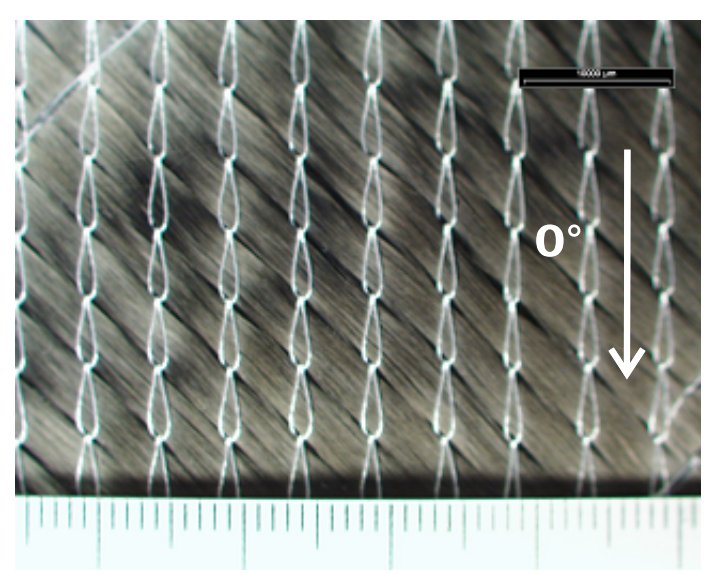

(a)

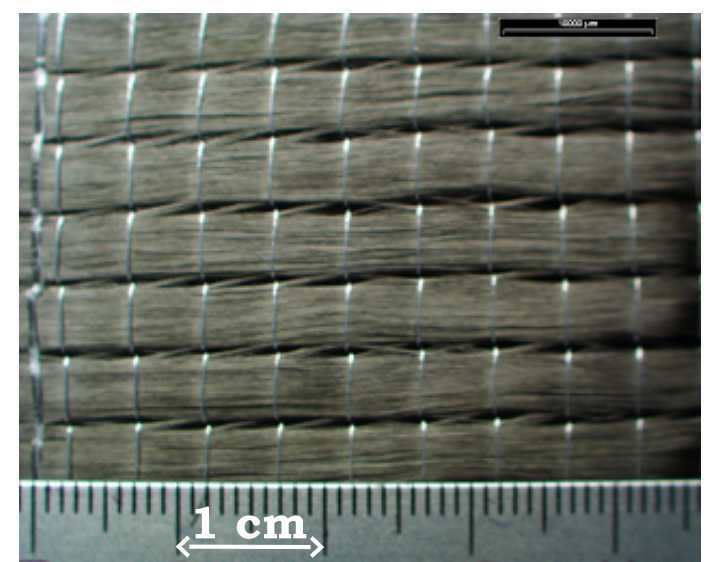

(b)

Figure 2: Picture of a quadriaxial $N C 2$ ([-45,0,45,90]) made up of dry carbon UD fabrics (Tenax HTS $5131 / 24 \mathrm{k}$ tows $150 \mathrm{~g} / \mathrm{m}^{2}$ ), stitching pitch $5 \mathrm{~mm} \times 5 \mathrm{~mm}:$ (a) $-45^{\circ}$ face and (b) $90^{\circ}$ face. 


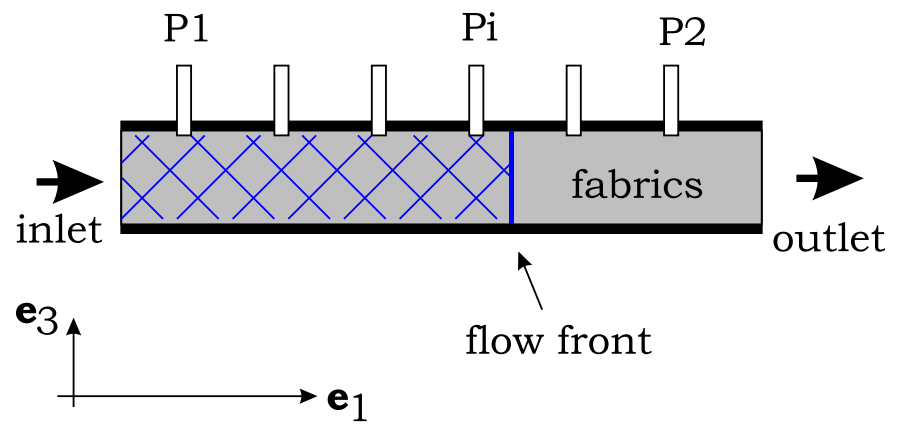

(a)

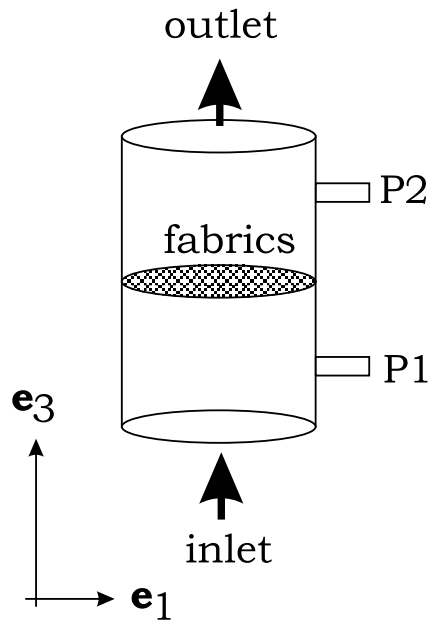

(b)

Figure 3: Schematic of (a) longitudinal, and (b) transverse permeability measurements. 


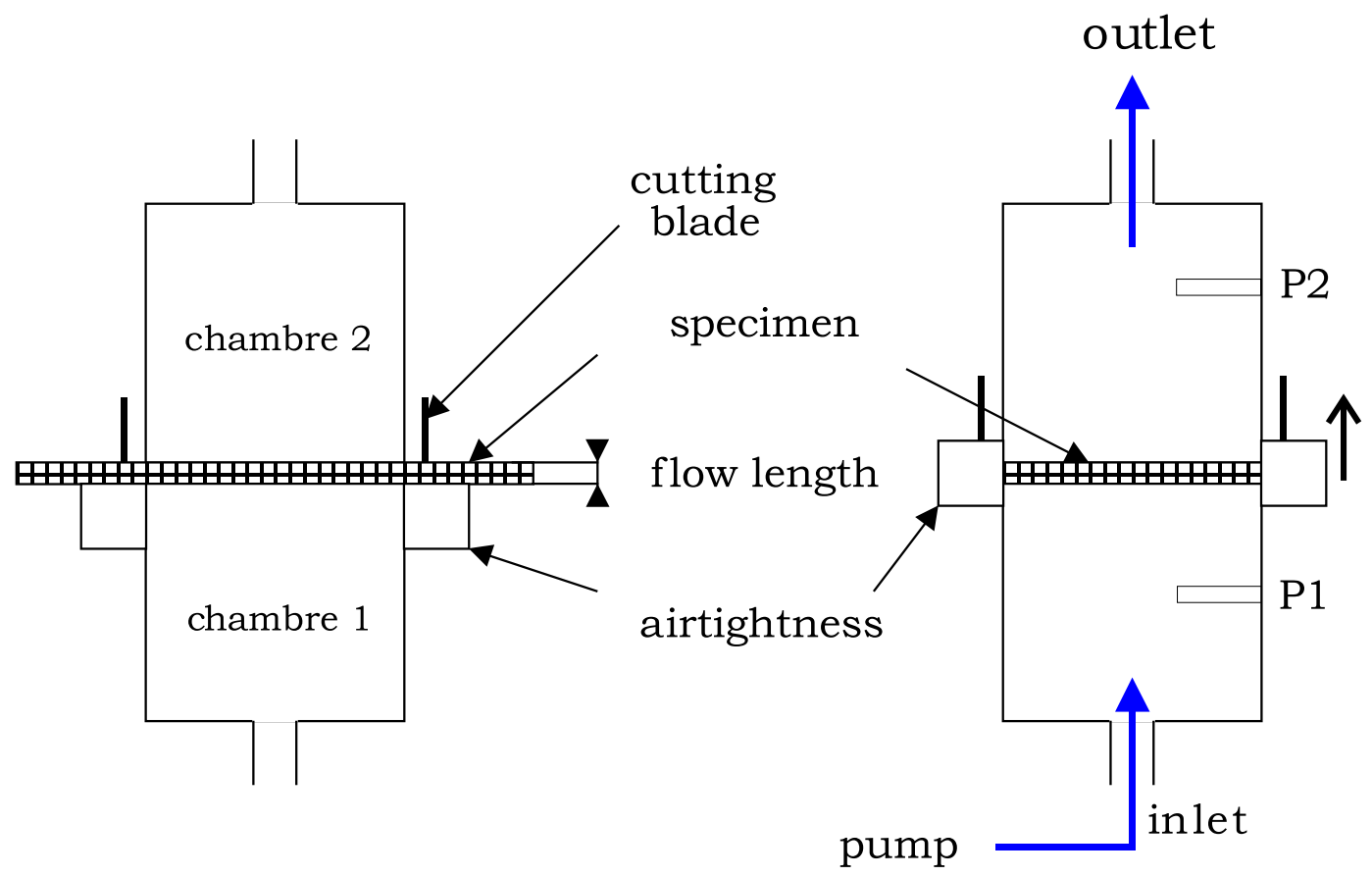

(a)

(b)

Figure 4: Schematic of the through-thickness permeability measurement device, in the 2 main stages of the protocol : (a) cutting stage, and (b) measurement stage. 


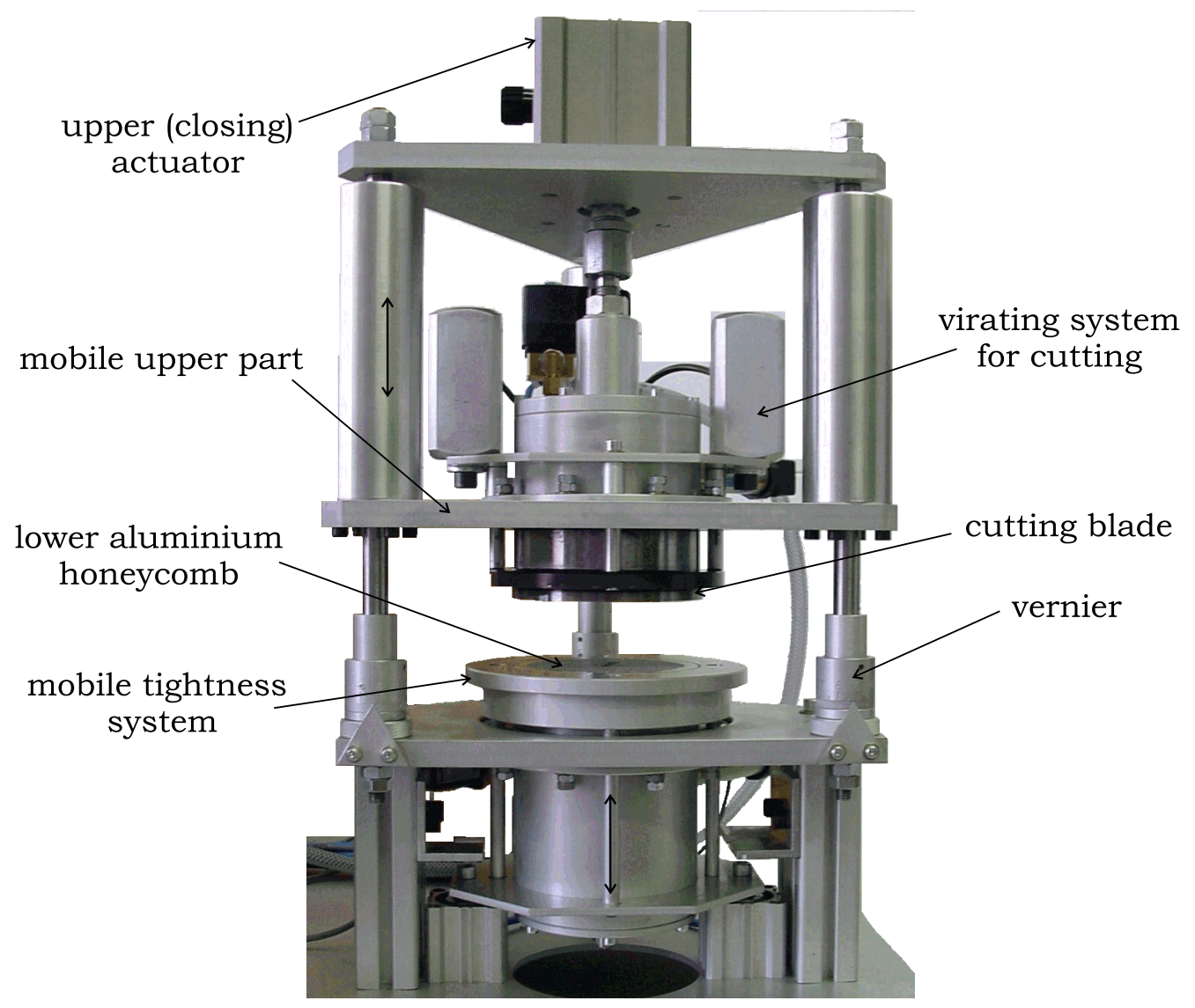

(a)

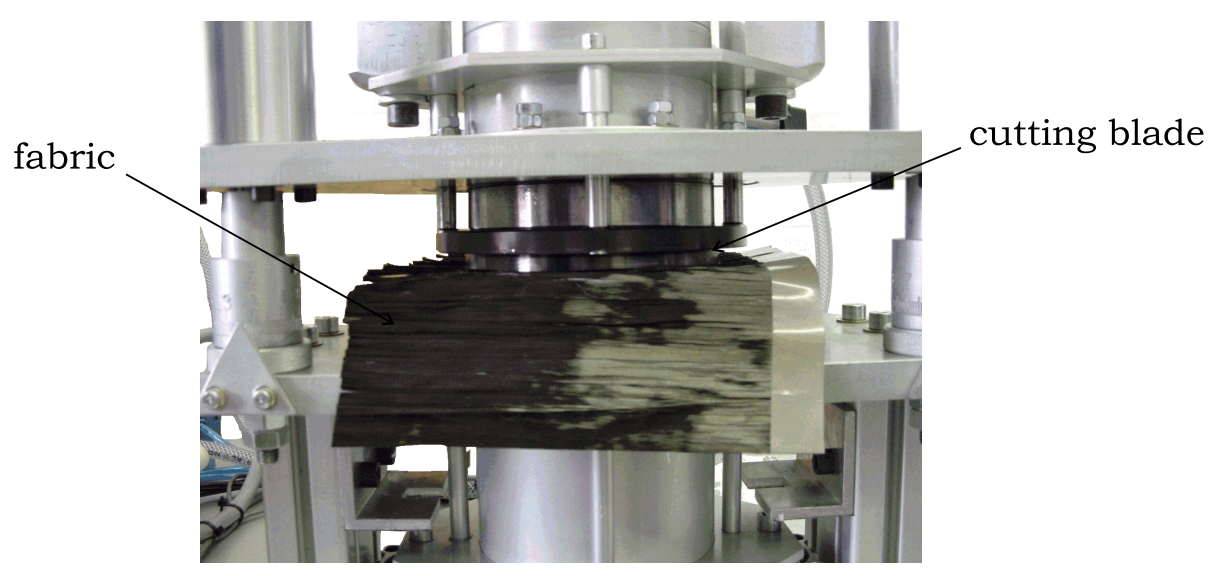

(b)

Figure 5: Apparatus designed for transverse permeability measurements : (a) description, (b) cutting stage prior to measurements . 


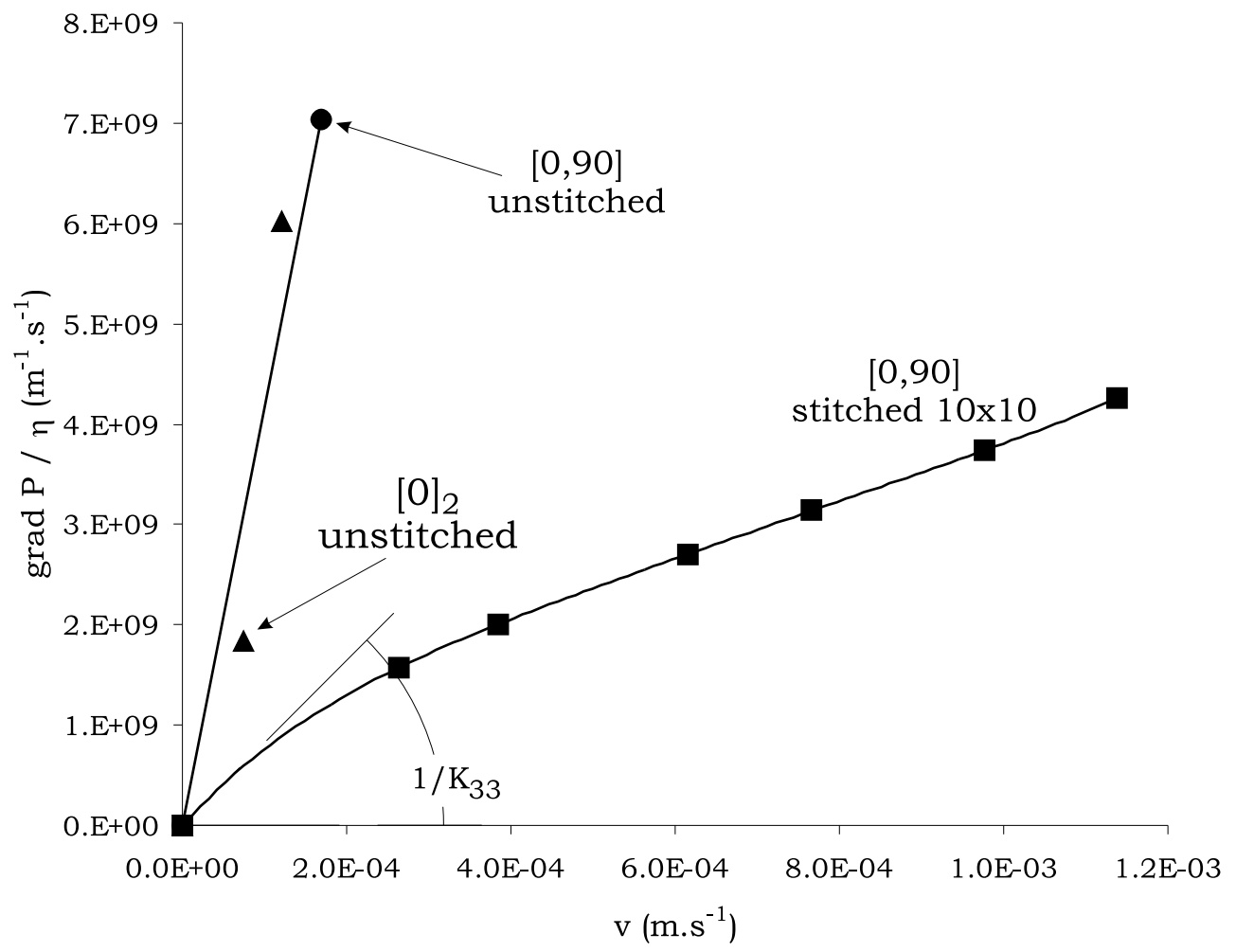

Figure 6: Resistance of fabrics to the fluid flow versus the fluid velocity for fabrics $2 \times$ $267 \mathrm{~g} / \mathrm{m}^{2}:[0]_{2}$ unstitched, $[0,90]$ unstitched, and $[0,90]$ stitched $10 \mathrm{~mm} \times 10 \mathrm{~mm}$ 


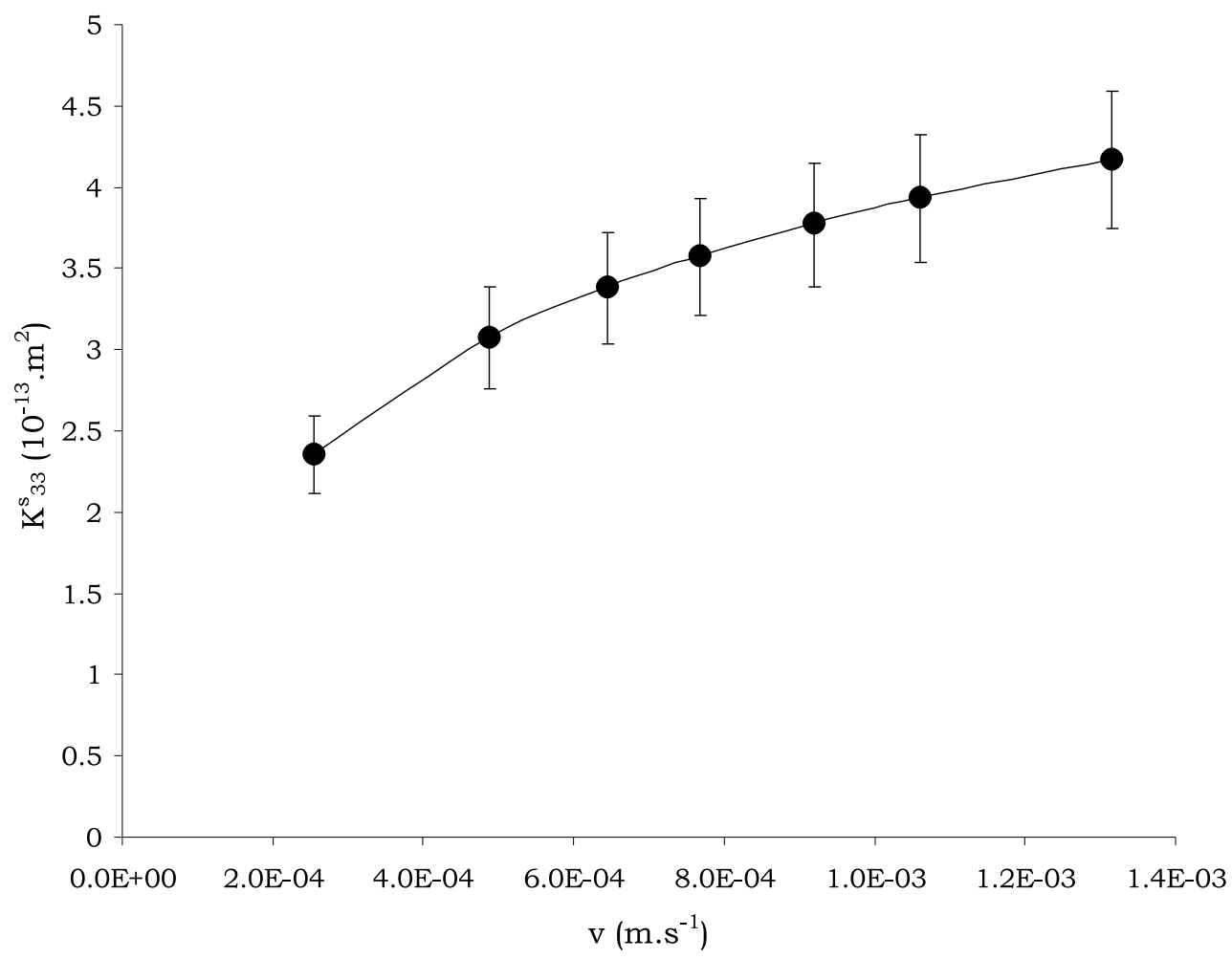

Figure 7: Secant permeability measurements $\left(K_{33}^{s}\right)$ versus the fluid velocity (v) for a biaxial $[0,90] N C 22 \times 267 \mathrm{~g} / \mathrm{m}^{2}$ stitching pitch $10 \mathrm{~mm} \times 10 \mathrm{~mm}$ 


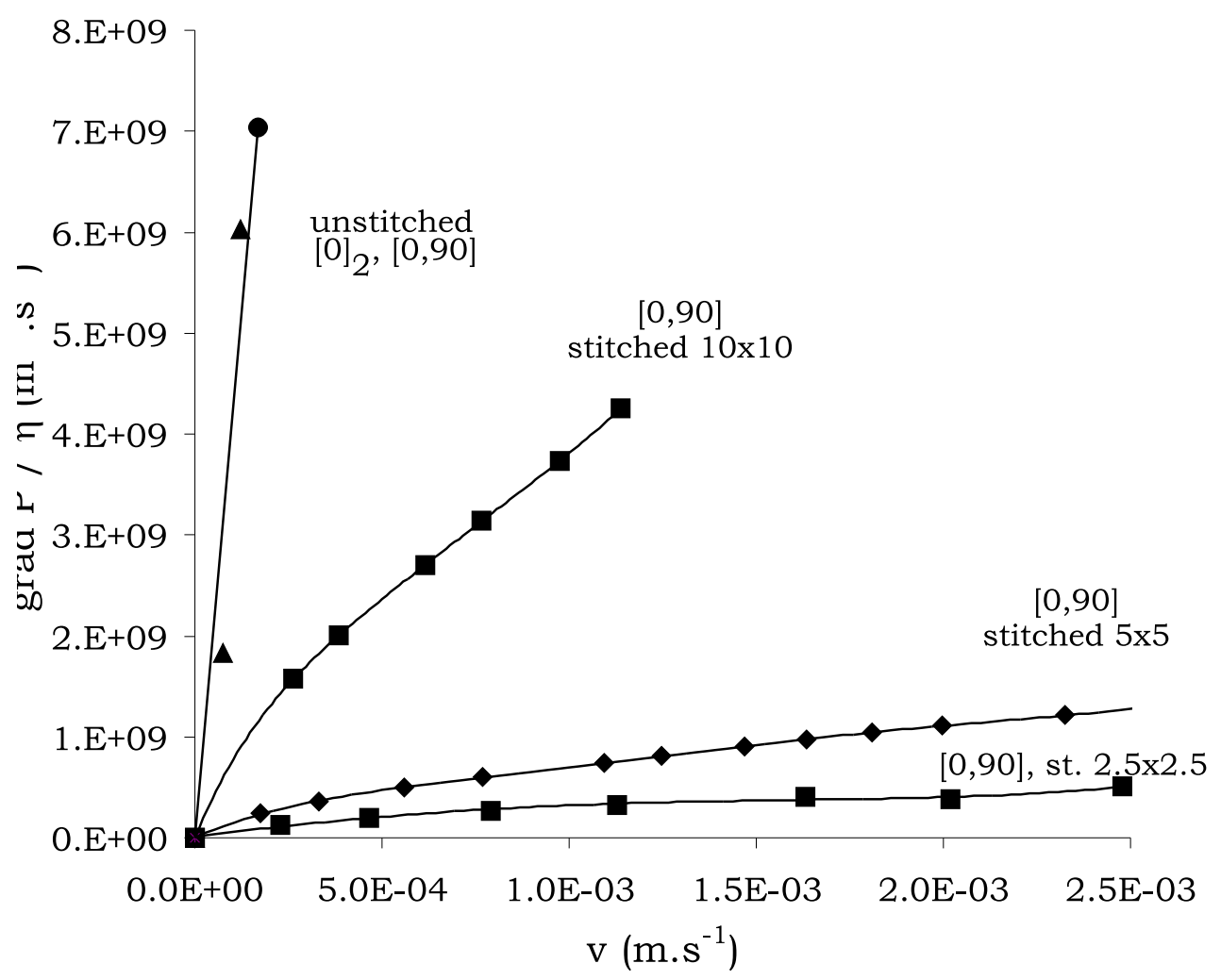

Figure 8: Resistance to the fluid flow versus the fluid velocity for fabrics $2 \times 267 \mathrm{~g} / \mathrm{m}^{2}$ : $[0]_{2}$ unstitched, biaxial $[0,90]$ unstitched and stitched $10 \mathrm{~mm} \times 10 \mathrm{~mm}, 5 \mathrm{~mm} \times 5 \mathrm{~mm}$, and $2.5 \mathrm{~mm} \times 2.5 \mathrm{~mm}$. 


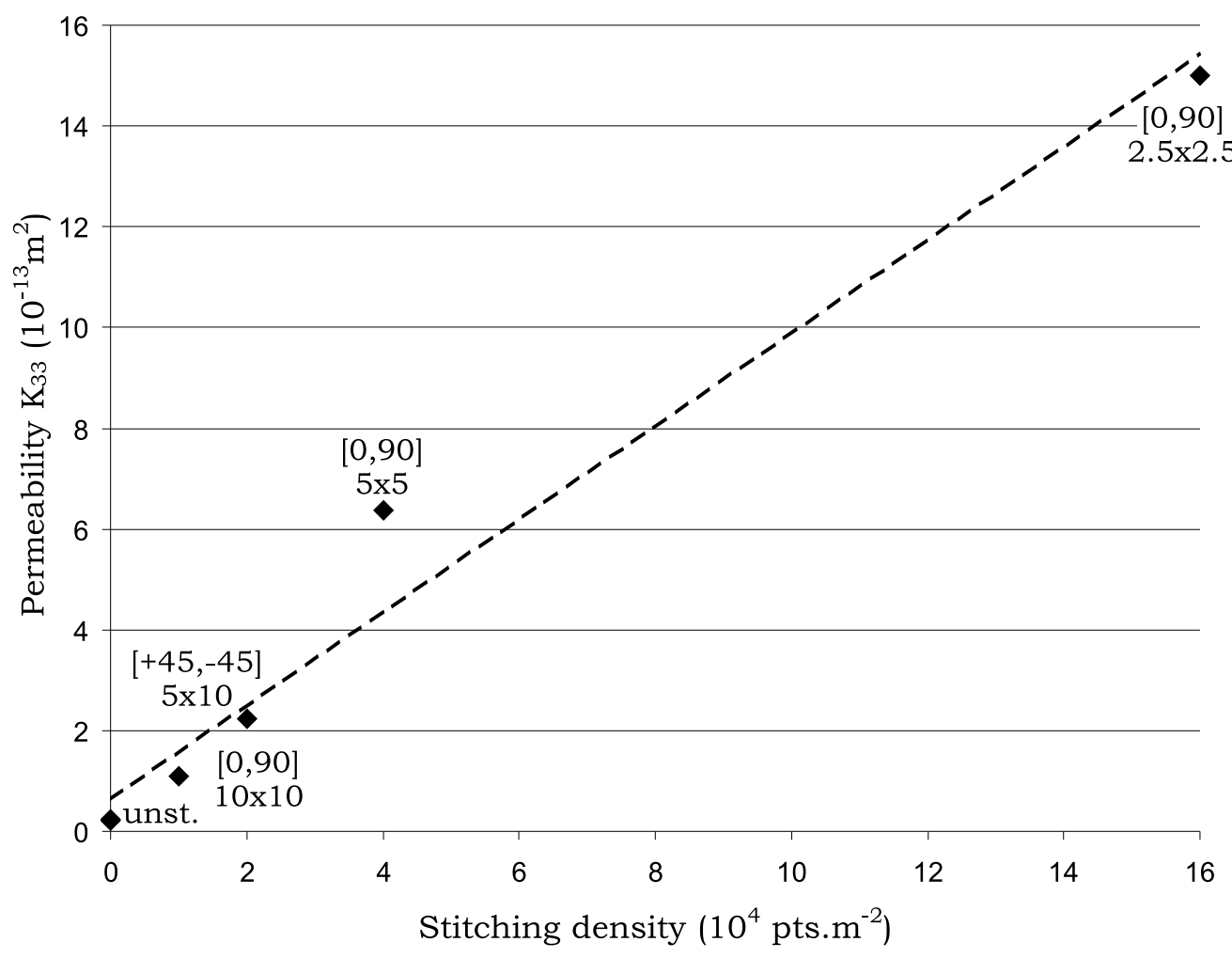

Figure 9: Permeability of the materials tested $v s$ their stitching density. 


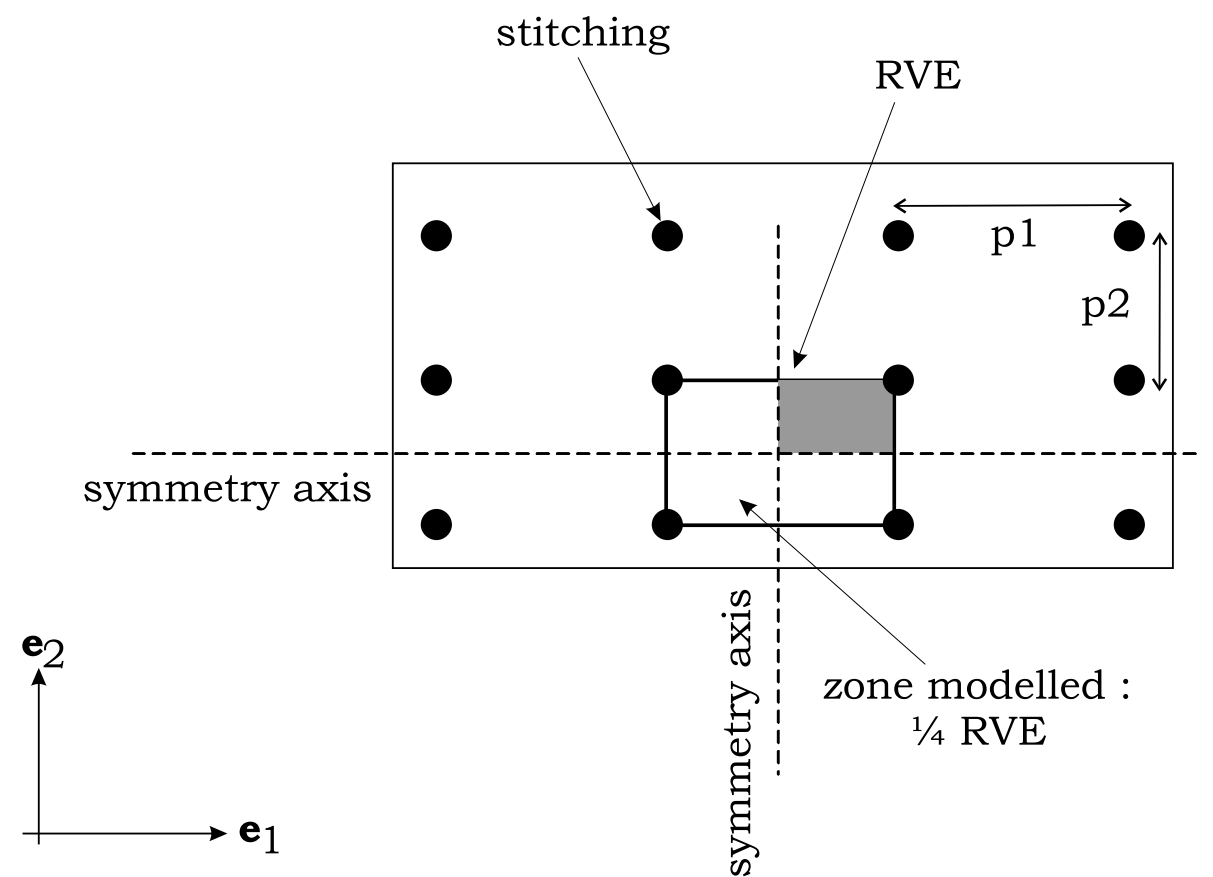

Figure 10: Representative Volume Element for the biaxial NC2 permeability simulation. 


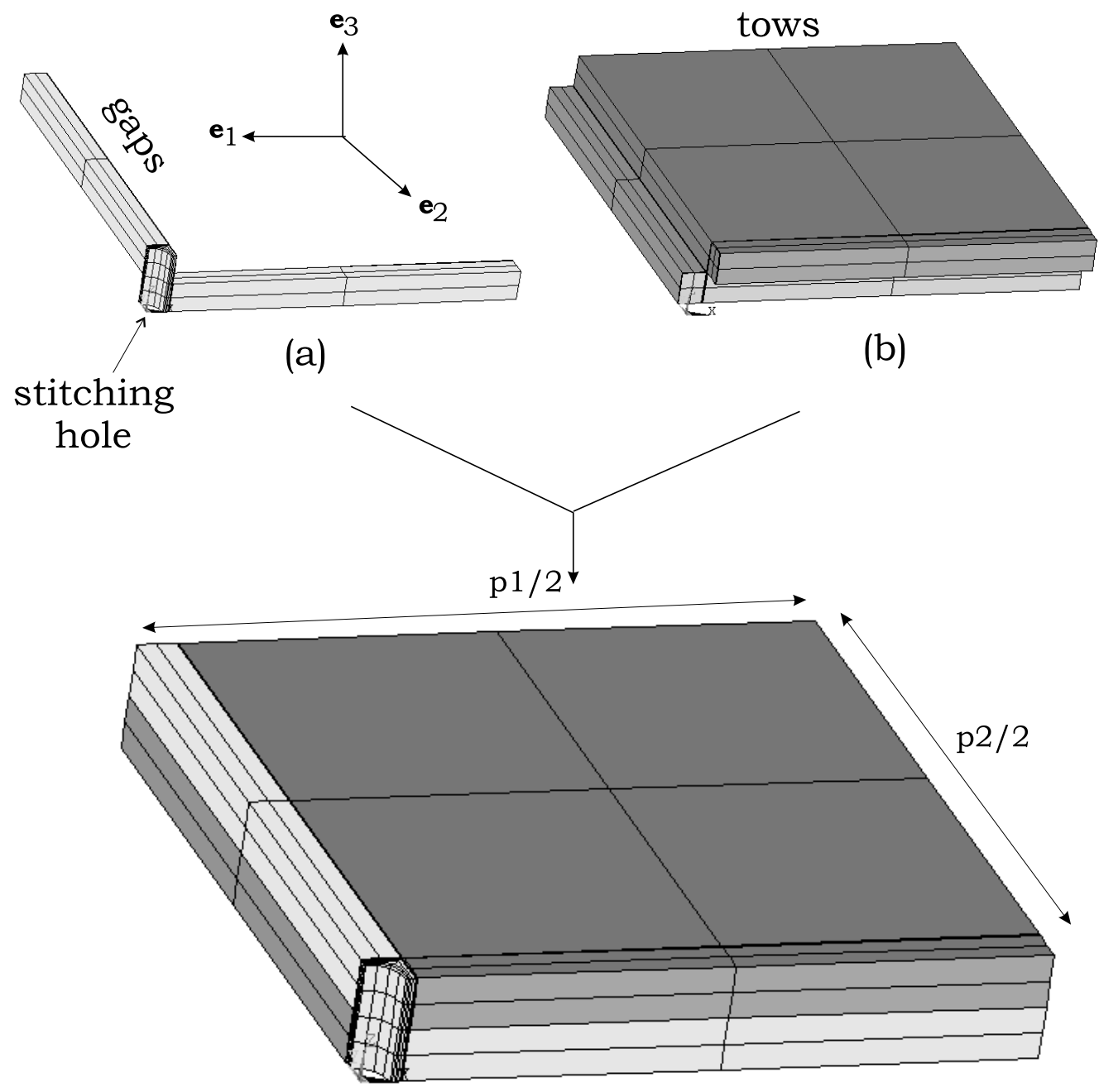

(c)

Figure 11: RVE mesh used for FE simulations : (a) mesh of the gaps with fluidic elements, (b) mesh of the tows with solid porous elements, and (c) complete mesh of the RVE. 


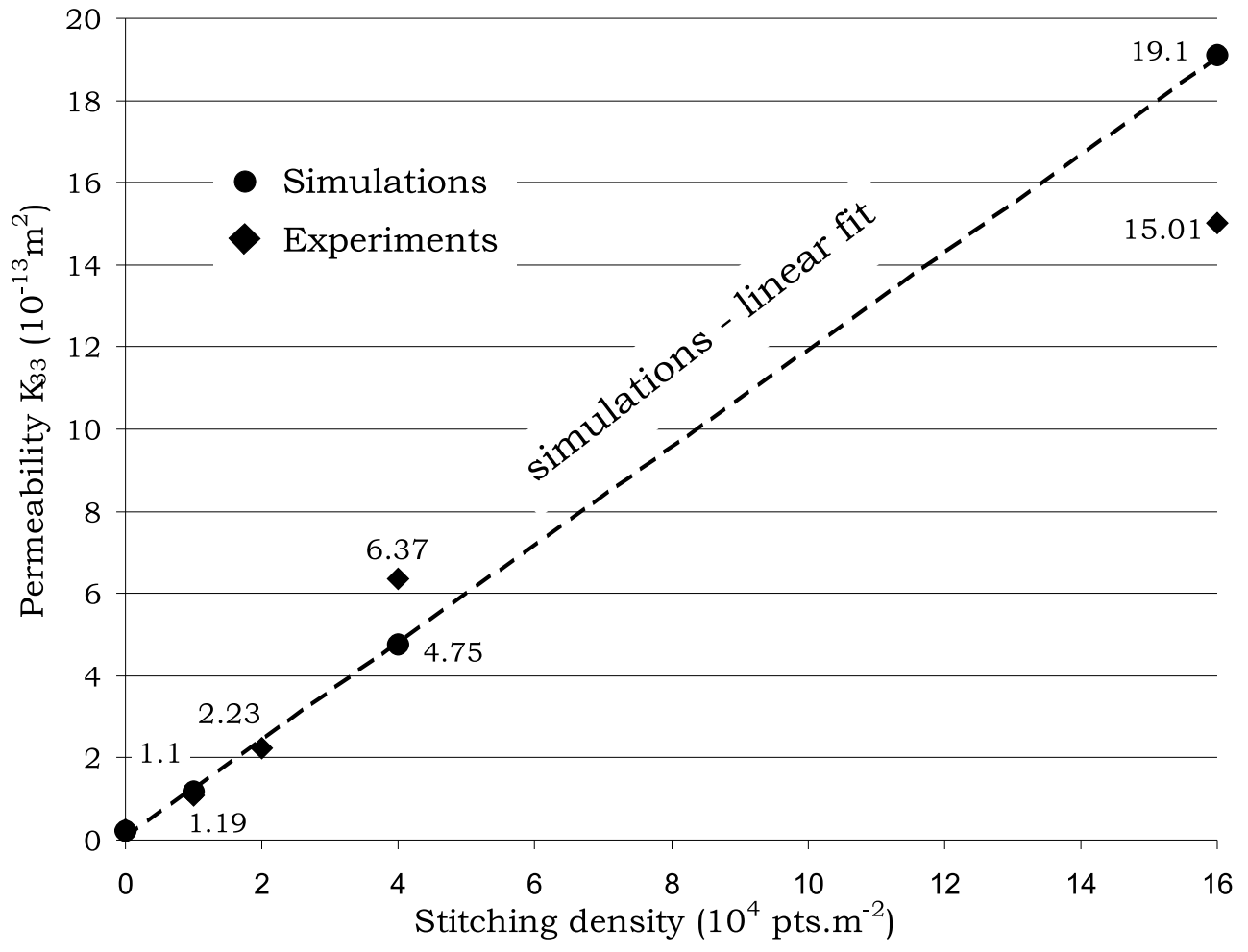

Figure 12: Permeability predicted and measured, for the materials tested, $v s$ their stitching density. 


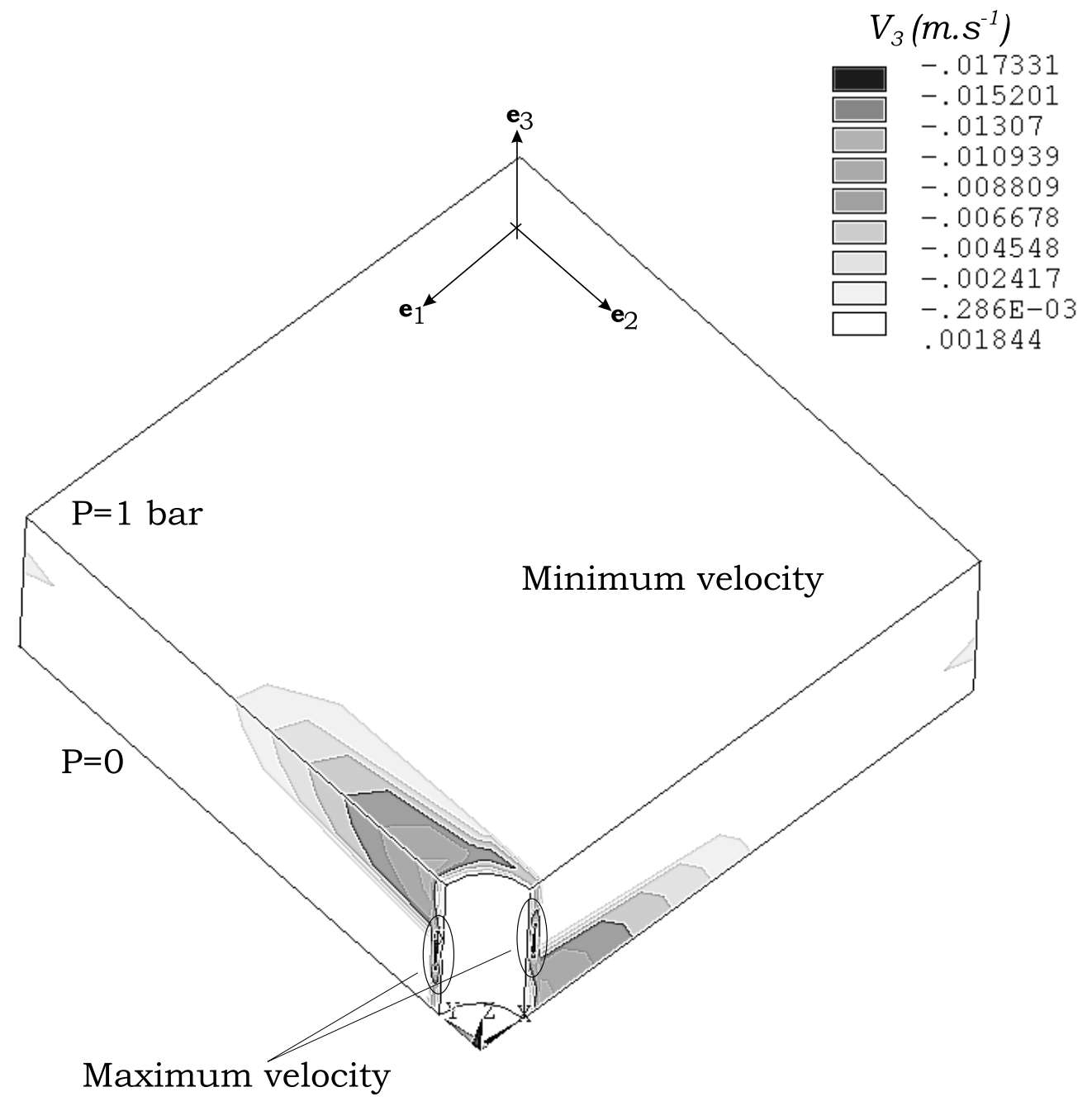

Figure 13: Distribution of the transverse fluid velocity $\left(V_{3}\right)$ in $m \cdot s^{-1}$ for the $[0,90] N C 2$ $5 \mathrm{~mm} \times 5 \mathrm{~mm}$ stitching pitch. 